\title{
Costo-efectividad de las cintas de uretra media comparada con el tratamiento convencional de la incontinencia urinaria femenina de esfuerzo en Colombia
}

\section{Cost-effectiveness of Tension-free Mid-urethral Tapes Compared with Retropubic Colposuspension and Pubovaginal Sling in Patients with Female Stress Urinary Incontinence in Colombia}

Hugo Enrique López-Ramos ${ }^{1}$ Herney Andrés García-Perdomo ${ }^{2}$ Víctor Prieto Martínez ${ }^{3} \quad$ Juan Prada ${ }^{4}$ Diego Rosselli ${ }^{5}$

\footnotetext{
1 Departamento de Urología, Hospital Universitario San Ignacio, Pontificia Universidad Javeriana, Bogotá, Colombia

2 Departamento de Urología, Universidad del Valle, Santiago de Cali, Colombia

${ }^{3}$ Departamento de Economía, Universidad Nacional de Colombia,

Bogotá, Colombia

${ }^{4}$ Departamento de Urología, Pontificia Universidad Javeriana, Bogotá, Colombia

${ }^{5}$ Departamento de Epidemiología, Pontificia Universidad Javeriana, Bogotá, Colombia

Urol Colomb 2020;29:141-147.
}

\author{
Address for correspondence Hugo Enrique López-Ramos, MD, \\ Department of Urología, Unidad de Urología, Hospital Universitario \\ San Ignacio, Pontificia Universidad Javeriana, Carrera 7a, 62/40, \\ Bogotá, Colombia (e-mail: hugolopez88@yahoo.es).
}

\section{Resumen}

Palabras Clave

- análisis costobeneficio

- incontinencia urinaria femenina de esfuerzo

- cintas de uretra media

- colposuspensión retropúbica

- cabestrillo pubovaginal

- árbol de decisión

- colombia
Objetivo Estimar la costo-efectividad de las cintas de uretra media comparada con la colposuspensión retropúbica y el cabestrillo pubovaginal en pacientes con incontinencia urinaria femenina de esfuerzo en Colombia.

Métodos Se construyó un árbol de decisión donde se comparó las cintas de uretra media con la colposuspensión retropúbica y el cabestrillo pubovaginal en el tratamiento quirúrgico de la incontinencia urinaria femenina de esfuerzo. La perspectiva fue la del tercer pagador incluyendo todos los costos directos. Todas las cifras monetarias se expresaron en pesos colombianos de 2019. La unidad de resultado fue la mejoría clínica definida como paciente continente o seca. Los datos de efectividad y seguridad se extrajeron de la literatura. Se calculó la razón de costoefectividad incremental. Se realizaron análisis de sensibilidad univariados y probabilísticos para los costos, efectos y supuestos del modelo.

Resultados Los resultados del modelo indican que el costo por un caso adicional de mejoría clínica del cabestrillo comparado con la colposuspensión fue de \$14 452753 received

December 13, 2019

accepted

January 13, 2020
DOI https://doi.org/

10.1055/s-0040-1708543.

ISSN 0120-789X.

e ISSN 2027-0119.
Copyright ( 2020, Sociedad Colombiana License terms de Urología. Publicado por Thieme Revinter Publicações Ltda., Rio de Janeiro, Brazil. Todos los derechos reservados.

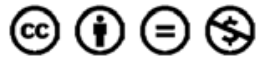




\author{
Abstract \\ Keywords \\ - cost-effectiveness \\ - tension-free mid- \\ urethral tapes \\ - retropubic \\ colposuspension \\ - pubovaginal sling \\ - female stress \\ urinary \\ incontinence \\ - decision tree model \\ - colombia
}

(4314 USD). El costo por un caso adicional de mejoría clínica de las cintas comparadas con el cabestrillo fue de $\$ 8098875$ (2417 USD).

Conclusión Desde el punto de vista económico, bajo los supuestos del modelo y desde el punto de vista del tercer pagador, las cintas de uretra media para el tratamiento de mujeres con incontinencia urinaria de esfuerzo, son costo-efectivas para Colombia. Los resultados fueron sensibles a los costos de los procedimientos quirúrgicos y a la efectividad de los mismos.

Objective To estimate the cost-effectiveness of mid-urethral tapes compared with retropubic colposuspension and pubovaginal sling in patients with female stress urinary incontinence in Colombia.

Methods A decision tree model was developed to compare tension-free mid-urethral tapes with retropubic colposuspension and pubovaginal sling for the treatment of female stress urinary incontinence from a third-party payer perspective, including all direct costs. All monetary figures are expressed in Colombian pesos for 2019. The unit of outcome was clinical improvement defined as dry or continent patient. The effectiveness and safety data were extracted from a systematic literature search. We calculated the incremental cost-effectiveness ratio. Univariate sensitivity analyzes were performed.

Results Model results indicate that if the willingness to pay for a case of additional improvement is greater than $\$ 8098875$ Colombian pesos (2417 USD), tension-free mid-urethral tapes represent the best alternative in terms of cost-effectiveness. These results were sensitive to the likelihood of clinical improvement of tension-free midurethral tapes.

Conclusion From the economic point of view, under the assumptions of the model and from a third-party payer perspective, mid-urethral tapes for the treatment of women with stress urinary incontinence, are cost-effective in Colombia. The results were sensitive to the costs and the effectiveness of surgical procedures.

\section{Introducción}

La incontinencia urinaria de esfuerzo está asociada a considerables costos económicos y perjuicios para las pacientes, afectando todos los aspectos de la calidad de vida, incluidas las dimensiones física, social, ocupacional, doméstica y sexual. ${ }^{1}$ Aunque la estimación de su prevalencia varía ampliamente entre 5 years 30\%, Luber reportó una prevalencia estimada de $30 \%$ en mujeres de 30 a 60 años de edad. $^{2}$

Los altos costos relacionados con el cuidado y los efectos secundarios superan el $2 \%$ de los gastos en salud en los Estados Unidos, haciendo que este sea un problema de salud pública. ${ }^{3}$

El manejo quirúrgico constituye el abordaje estándar para las mujeres con incontinencia urinaria de esfuerzo en quienes han fallado las estrategias de manejo conservadoras como cambios en el estilo de vida, terapia física, regímenes de micción por horario y terapias de retroalimentación. ${ }^{4}$ El objetivo es restaurar la función del tracto urinario femenino de la forma más parecida posible a lo normal, con la menor morbilidad a corto y largo plazo.
Aunque se han reportado más de un centenar de técnicas quirúrgicas diferentes, la técnica quirúrgica ideal, - es decir, un procedimiento simple, económico, fácil de aprender y de realizar, mínimamente invasivo, de alta y durable eficacia y sin morbilidad a largo plazo ni secuelas funcionales, - todavía no existe.

Durante las últimas décadas se han producido importantes cambios en el tratamiento de esta patología, basados en un mejor conocimiento de la fisiopatología del piso pélvico y de los trastornos de la micción, llevando a la introducción de nuevas técnicas quirúrgicas menos invasivas y por ende, menos incapacitantes para las pacientes.

El tratamiento convencional ha sido el manejo quirúrgico con diferentes técnicas, algunas de las cuales implican incisiones abdominales como la colposuspensión retropúbica descrita por Burch en $1961^{5}$; o el cabestrillo pubovaginal, abordaje combinado abdominal y vaginal que requieren un tiempo de hospitalización de dos o tres días.

Desde hace algunos años se realiza un nuevo procedimiento quirúrgico que consiste en la colocación de 
una cinta de polipropileno, libre de tensión por debajo de la uretra media. Este procedimiento es de carácter ambulatorio, realizado en menor tiempo quirúrgico y sin necesidad de incisión abdominal.

Tanto la colposuspensión retropúbica como el cabestrillo pubovaginal tienen evidencia sólida de eficacia a largo plazo, con seguimiento mayor a 10 años y tasas de éxito superiores al $80 \%$. Sin embargo, ninguna de estas técnicas es mínimamente invasiva, y además presentan síntomas miccionales hasta en un $25 \%{ }^{6}$

Desde los primeros reportes del grupo de Ulmsted, ${ }^{7}$ la colocación de la cinta transvaginal libre de tensión (TVT), -la primera cinta de polipropileno para uretra media- ha sido una de los procedimientos más realizados en el mundo, debido a la facilidad de su aplicación, así como a las tasas de éxito en el corto plazo. En 2008, Nilsson reportó resultados con un seguimiento de 11 años demostrando que el $90 \%$ de las mujeres operadas estaban objetivamente curadas sin ningún efecto tardío significativo. ${ }^{8}$

El objetivo del presente estudio fue estimar la costoefectividad de las cintas de uretra media comparadas con la colposuspensión retropúbica y el cabestrillo pubovaginal en pacientes con incontinencia urinaria femenina de esfuerzo en Colombia.

\section{Métodos}

Se realizó un estudio de tipo evaluación económica en el que se construyó un modelo (-Figura 1) para simular la toma de una decisión, por parte de un médico tratante, en una cohorte hipotética de pacientes de sexo femenino, de 50 años de edad con incontinencia urinaria de esfuerzo primaria que requiere y desea tratamiento quirúrgico, susceptible de ser manejada con cualquiera de las siguientes tres opciones terapéuticas:

- Colposuspensión retropúbica

- Cabestrillo pubovaginal

- Cinta de uretra media

Se escogió la edad de 50 años, porque es en la que se presenta la mayor prevalencia de incontinencia urinaria femenina. ${ }^{2}$ El análisis fue conducido desde una perspectiva de un tercero pagador con un horizonte a cinco años y se descontaron los costos y efectos a una tasa de $3 \%$.

\section{Supuestos}

Cuando la cirugía inicial fracasa, se asumió que en el $80 \%$ de los casos (70-90\%) las pacientes en conjunto con su médico tratante escogerán la realización de un segundo procedimiento.

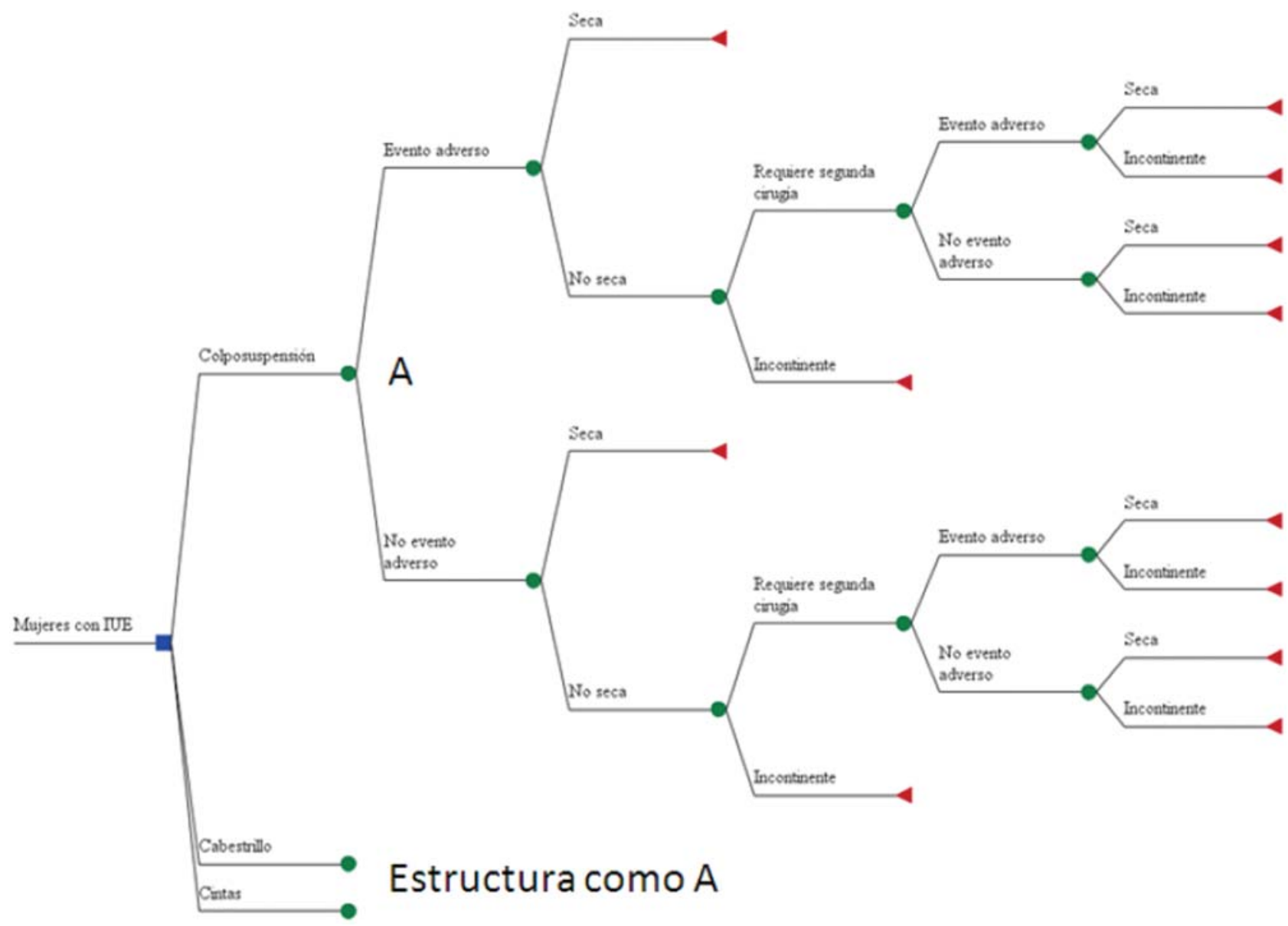

Fig. 1 Árbol de decisión para tres técnicas quirúrgicas en el manejo de la incontinencia urinaria femenina de esfuerzo. 
Cuadro 1 Datos de efectividad y seguridad utilizados en el modelo de evaluación económica

\begin{tabular}{|l|l|l|l|l|}
\hline \multirow{2}{*}{ Descripción de la variable } & \multicolumn{2}{|l|}{$\begin{array}{l}\text { Análisis de sensibilidad } \\
\text { determinístico }\end{array}$} & $\begin{array}{l}\text { Análisis de sensibilidad } \\
\text { probabilístico }\end{array}$ & \multirow{2}{*}{ Referencia } \\
\cline { 2 - 5 } & Caso base & Rango & Distribución y parámetros & \\
\hline Probabilidad de cura con la colposuspensión & 0,73 & $0,64-0,77$ & Beta $(2410,891)$ & AUA $^{11}$ \\
\hline Probabilidad de cura con el cabestrillo & 0,82 & $0,67-0,93$ & Beta $(1874,411)$ & AUA $^{11}$ \\
\hline Probabilidad de cura con la cinta de uretra media & 0,88 & $0,82-0,98$ & Beta $(6279,856)$ & Ogah $^{9}$ \\
\hline Probabilidad de segunda cirugía (cuando falla 1) & 0,8 & $0,70-0,90$ & Uniforme $(0,70-0,90)$ & Ver texto $^{11}$ \\
\hline Probabilidad de erosión de la cinta & 0,01 & $0,005-0,05$ & Beta $(71,7051)$ & Levin $^{10}$ \\
\hline Probabilidad de evento adverso post-colposuspensión & 0,32 & $0,28-0,36$ & Beta $(210,445)$ & Albo $^{14}$ \\
\hline Probabilidad de evento adverso post-cabestrillo & 0,48 & $0,44-0,52$ & Beta $(314,341)$ & Albo $^{14}$ \\
\hline
\end{tabular}

Después de la colocación de cualquiera de las cintas, la probabilidad de erosión es del $1 \%^{9}$ con un rango que varía del $0,05 \%$ al $5 \%$. Se asume que las erosiones de la cinta son reparadas con cirugía vaginal, logrando que el $90 \%$ de las pacientes persistan continentes después del procedimiento.

El evento adverso de mayor frecuencia tanto para la colposuspensión retropúbica como para el cabestrillo pubovaginal de acuerdo a la literatura es la infección de vías urinarias. Esta se presenta en el 48\% después del cabestrillo pubovaginal y en el $32 \%$ después de la colposuspensión retropúbica. $^{10}$

\section{Datos de Efectividad y Seguridad}

La probabilidad de cura objetiva para la técnicas de la colposuspensión retropúbica y el cabestrillo pubovaginal fue tomada del meta-análisis realizado por el panel de la guía de la Asociación Americana de Urología (AUA), ${ }^{11}$ donde se presentan los resultados con un tiempo mayor a 48 meses. El desenlace evaluado fue la cura objetiva definida como paciente seca. La probabilidad de curación a 48 meses para la colposuspensión retropúbica fue de 73\% (IC del 95\%: 64 a 77\%), y para el cabestrillo pubovaginal fue de $82 \%$ (IC del 95\%: 67 a 93\%).

Estos hallazgos son similares a los publicados por Lapitan, en la revisión Cochrane año 2005 para colposuspensión retropúbica, ${ }^{12}$ donde incluyó 39 ensayos para un total de
3301 mujeres, encontrando una probabilidad de curación del $70 \%$ a 5 años.

Así mismo, los datos del cabestrillo son similares a los publicados por Rehman en la revisión Cochrane año 2011, ${ }^{13}$ que incluyó 26 estudios con 2284 mujeres, encontrando una probabilidad de curación del $81 \%$.

La probabilidad de cura objetiva para la cinta de uretra media fue tomada de la revisión Cochrane año $2009^{9}$ que incluyó 62 estudios con 7101 mujeres, reportando una probabilidad de curación objetiva del $88 \%$. No se tomaron los datos del meta-análisis de la AUA porque la revisión tuvo un punto de corte al año 2005 que no incluía la mayoría de los estudios de cintas tipo TOT.

En el Cuadro 1 se presentan los datos que fueron insumo para la construcción del modelo.

\section{Uso de Consumo de Recursos y Costos}

Se construyó un caso tipo basado en un protocolo de manejo de una paciente con incontinencia urinaria de esfuerzo desde que asiste a la valoración inicial con el médico general en el primer nivel de atención, hasta su último control postoperatorio por el médico especialista que realiza la intervención quirúrgica. El protocolo de manejo fue evaluado por un consenso de expertos constituido por tres especialistas urólogos.

Cuadro 2 Costos totales empleados en la evaluación económica

\begin{tabular}{|l|l|l|l|l|}
\hline \multirow{2}{*}{ Descripción de la variable } & \multicolumn{2}{|l|}{ Análisis de sensibilidad determinístico } & $\begin{array}{l}\text { Análisis de sensibilidad } \\
\text { probabilístico }\end{array}$ & Fuente \\
\cline { 2 - 5 } & Caso base & Rango & Distribución y parámetros & ISS 2001 \\
\hline Colposuspensión & $\$ 1459482,04$ & $\$ 1151294,24-\$ 1767668,53$ & $\begin{array}{l}\text { Gamma } \\
(2712586,2,42)\end{array}$ & ISS 2001 \\
\hline Cabestrillo & $\$ 1903191,54$ & $\$ 1506262,10-\$ 2300120,97$ & $\begin{array}{l}\text { Gamma } \\
(3581395,2,45)\end{array}$ & ISS 2001 \\
\hline Cintas de uretra media & $\$ 2276827,14$ & $\$ 1701170,32-\$ 2852483,69$ & $\begin{array}{l}\text { Gamma } \\
(3522317,2,01)\end{array}$ & $\begin{array}{l}\text { Gamma } \\
(1408547,2,31)\end{array}$ \\
\hline Extrusión de la cinta & $\$ 791655,13$ & $\$ 617031,41-\$ 966277,28$ & $\begin{array}{l}\text { Gamma } \\
(183743,2,22)\end{array}$ & ISS 2001 \\
\hline Costo de la infección urinaria & $\$ 107544,58$ & $\$ 114265,94-\$ 193674,0$ & &
\end{tabular}


Análisis de costo-efectividad Paciente con IUE

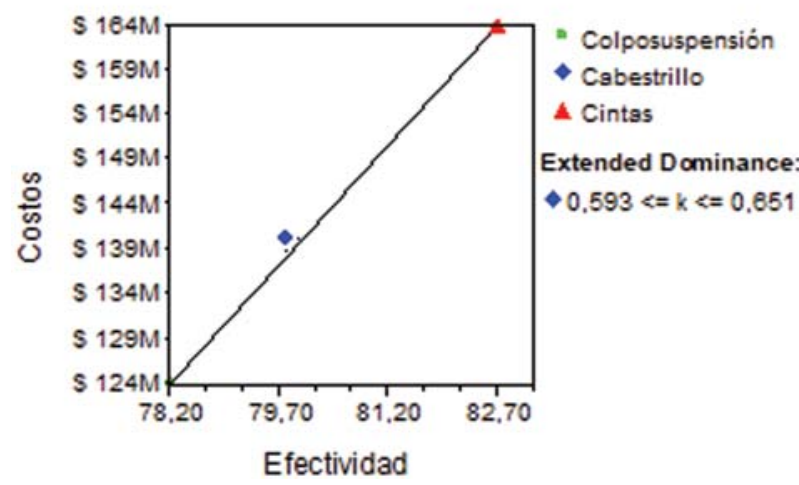

Fig. 2 Plano de costo-efectividad, para una cohorte de 100 pacientes.

Para la estimación de los costos se identificaron y cuantificaron los eventos generadores de costos, y posteriormente, se desarrolló una valoración de los mismos (ver Cuadro 2).

\section{Medicamentos}

El costo promedio de los medicamentos fue extraído de la base SISMED (Sistema integrado de información de precios de medicamentos) versión $2008,{ }^{15}$ administrada por la Comisión Nacional de Precios de Medicamentos. Los costos fueron ajustados a valores de 2011 según el incremento en el índice de precios al consumidor (IPC) del total nacional.

\section{Procedimientos}

Se adoptó como fuente de costos de los procedimientos los precios del Manual de Tarifas ISS (Instituto de Seguro Social) $2001^{16}$ con un incremento de $30 \%$, cifra que en los análisis de sensibilidad tomó valores de $20 \%$ por encima y por debajo como límites superior e inferior. El incremento porcentual y los valores para el análisis de sensibilidad, fueron calculados a través de un consenso con distintos prestadores de servicios de salud.

\section{Insumos}

Debido a que la información de suministros no se encuentra en ninguna fuente de información oficial, para la identificación de costos de los insumos, específicamente el valor de las cintas, se recurrió a valores de mercado y a catálogos de precios de proveedores presentados en diversos procesos de licitación, que obedecen a información pública. Para los análisis de sensibilidad se utilizó un rango de 20\% sobre el costo total de los insumos.

\section{Resultados}

Se calculó la razón de costo-efectividad incremental, definida como el cociente entre la diferencia de costos sobre la diferencia de efectos. Se realizaron análisis de sensibilidad univariados y probabilísticos para los costos, efectos y supuestos del modelo. Se graficó el diagrama de tornado y el diagrama de dispersión. También se construyó la curva de aceptabilidad.

\section{Análisis de Costo Efectividad}

Los resultados del estudio se muestran de manera gráfica en la - Figura 2 years la síntesis de los resultados se presenta en Cuadro 3.

Los resultados del modelo indican que el costo por un caso adicional de mejoría clínica del cabestrillo comparado con la colposuspensión fue de \$14 452 753. El costo por un caso adicional de mejoría clínica de las cintas comparadas con el cabestrillo fue de \$8 098875 .

\section{Análisis de Incertidumbre}

El diagrama de Tornado (-Fig. 3) mostró que la razón de costo-efectividad incremental es afectada principalmente por: el costo de las cintas de uretra media, la probabilidad de cura con las cintas de uretra media, el costo de la colposuspensión, la probabilidad de cura con la colposuspensión, la probabilidad de cura con el cabestrillo y la probabilidad de requerir una segunda cirugía. Dichas variables explican el $98 \%$ de la incertidumbre en la evaluación económica.

\section{Análisis de Sensibilidad Probabilístico}

Se realizó una simulación de Montecarlo con 10000 iteraciones. En ausencia de un umbral para Colombia, para determinar la costo-efectividad de las alternativas en evaluación, se asumió como posible disponibilidad a pagar por un caso adicional de mejoría clínica el PIB per cápita para Colombia año 2019, el cual era de $\$ 22281850^{17}$ representado en la -Figura 4 por la línea punteada. En el 99,98\% de las iteraciones, las cintas de uretra media son la mejor alternativa en términos de costo-efectividad.

Cuadro 3 Resultados del análisis de costo-efectividad

\begin{tabular}{|l|l|l|l|l|l|l|}
\hline Estrategia & Costos & $\begin{array}{l}\text { Costo } \\
\text { incremental }\end{array}$ & Efectividad & $\begin{array}{l}\text { Efectividad } \\
\text { incremental }\end{array}$ & $\begin{array}{l}\text { Costo } \\
\text { efectividad } \\
\text { promedio }\end{array}$ & $\begin{array}{l}\text { Razón de } \\
\text { costo-efectividad } \\
\text { incremental }\end{array}$ \\
\hline Colposuspensión & $\$ 167.700 .000,00$ & & 78,25 & & $\$ 2.141 .298$ & \\
\hline Cabestrillo & $\$ 189.800 .000,00$ & $\$ 22.100 .000,00$ & 79,8 & 1,55 & $\$ 2.380 .842$ & $\$ 14.452 .753$ \\
\hline Cintas & $\$ 213.200 .000,00$ & $\$ 245.700 .000,00$ & 82,7 & 2,9 & $\$ 2.581 .241$ & $\$ 8.098 .875$ \\
\hline
\end{tabular}




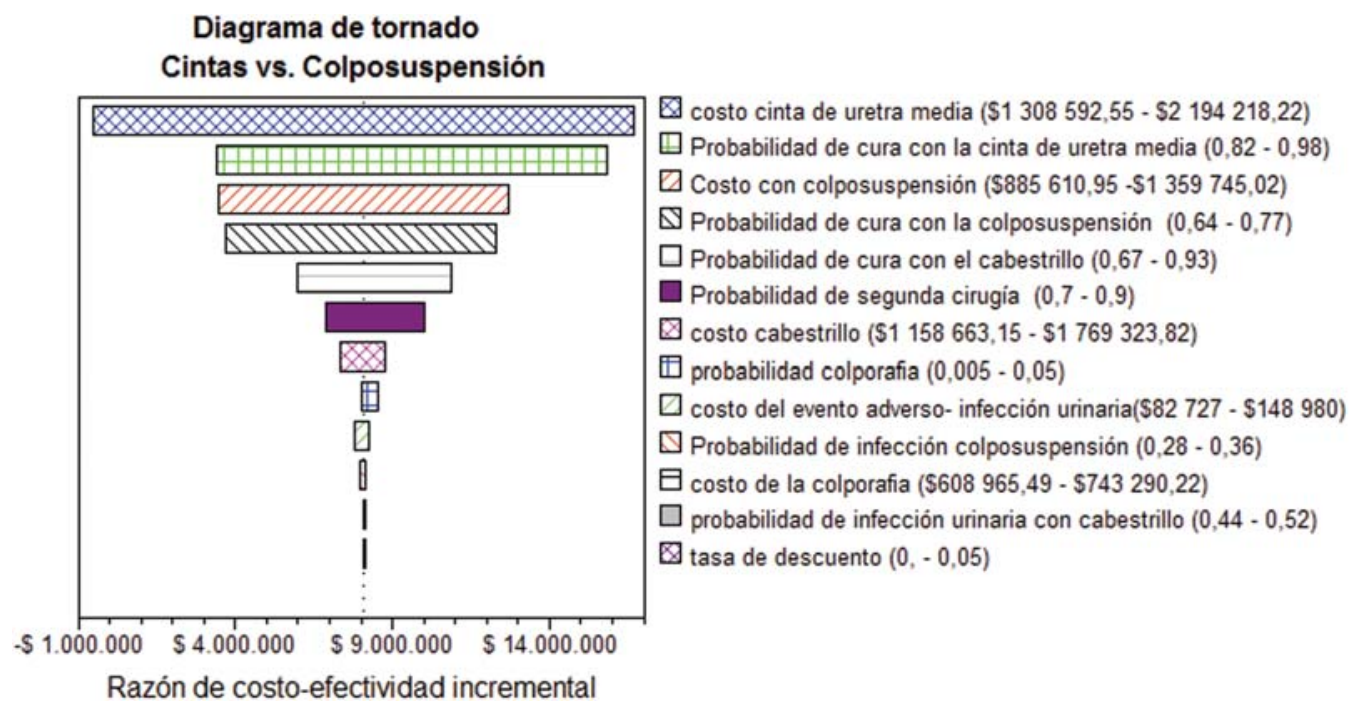

Fig. 3 Diagrama de Tornado.

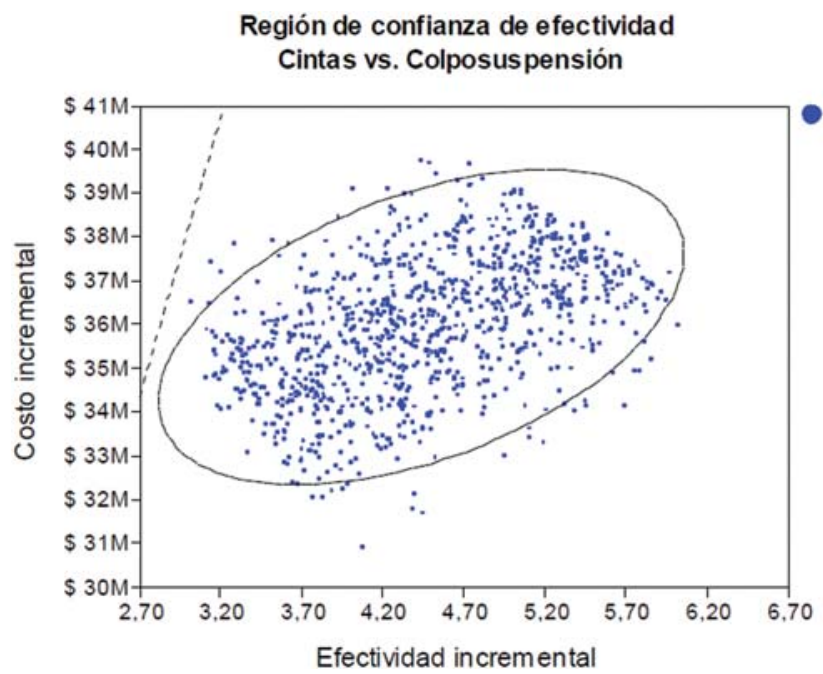

Fig. 4 Región de confianza de efectividad.

\section{Discusión}

Existen diversas técnicas para el manejo de la incontinencia urinaria de esfuerzo, siendo importante destacar que en los últimos años han surgido procedimientos menos invasivos y de carácter ambulatorio como las cintas de uretra media, incluso presentando tasas superiores de efectividad en la literatura. Por esta razón era necesario realizar una evaluación económica en el escenario colombiano.

No existe un estudio económico que permita evaluar la razón de costo-efectividad incremental de cada uno de los tratamientos en Colombia. El tema es de gran relevancia en el momento actual debido a que la disminución de los costos en la prestación de los servicios sin detrimento de la calidad, es la base del sistema de aseguramiento vigente.

Cuando se adoptan nuevas tecnologías quirúrgicas el análisis de costo-efectividad constituye una consideración crítica, siendo de ésta manera relevante para el urólogo, el ginecólogo, el médico general y el trabajador de la salud, poder utilizar la información provista por este tipo de estudios, brindándoles herramientas para una mejor toma de decisiones involucradas en la asignación de recursos.

\section{Futuras Investigaciones}

En Colombia no tenemos estimaciones de los AVAC - años de vida ajustados por calidad; para estimar calidad de vida de las mujeres con incontinencia urinaria de esfuerzo. Dado que las preferencias de los pacientes varían de acuerdo al acceso al servicio, a los sistemas de salud y a las condiciones socioculturales particulares de cada sociedad se recomienda la realización de estudios que permitan conocer el valor de los AVAC para incontinencia urinaria femenina en Colombia.

Para futuras evaluaciones económicas, se recomienda que las técnicas que se reporten en la literatura, se validen en estudios locales para darle mayor solidez a las evaluaciones económicas.

\section{Conclusión}

Desde el punto de vista económico, bajo los supuestos del modelo y desde el punto de vista del tercer pagador, las cintas de uretra media para el tratamiento de mujeres con incontinencia urinaria de esfuerzo, son costo-efectivas para Colombia. Los resultados fueron sensibles a los costos de los procedimientos quirúrgicos y a la efectividad de los mismos.

Si la disponibilidad a pagar por un caso de mejoría adicional es mayor que \$ 8098875 (2417 USD), las cintas de uretra media representan la mejor alternativa en términos de costo-efectividad. Por otra parte, si la disponibilidad a pagar es de \$14 452753 (4314 USD) por un caso de mejoría clínica adicional, el cabestrillo pubovaginal sería la mejor alternativa en términos de costo-efectividad. 
Conflicto de Intereses

Los autores declaran no tener ningún conflicto de intereses.

\section{Referencias}

1 Salonia A, Zanni G, Nappi RE, et al. Sexual dysfunction is common in women with lower urinary tract symptoms and urinary incontinence: results of a cross-sectional study. Eur Urol 2004; 45(05):642-648, discussion 648

2 Luber KM. The definition, prevalence, and risk factors for stress urinary incontinence. Rev Urol 2004;6(Suppl 3):S3-S9

3 Hu TW, Wagner TH, Bentkover JD, Leblanc K, Zhou SZ, Hunt T. Costs of urinary incontinence and overactive bladder in the United States: a comparative study. Urology 2004;63(03):461-465

4 Abrams P, Andersson KE, Birder L, et al. Recommendations of the International Scientific Committee: evaluation and treatment of urinary incontinence, pelvic organ prolapse and faecal incontinence. En: Abrams P, Cardozo L, Khoury S, et al, editors. Incontinence 4th ed. Plymouth, UK: Health Publications; 2009: 1767-854

5 Burch JC. Urethrovaginal fixation to Cooper's ligament for correction of stress incontinence, cystocele, and prolapse. Am J Obstet Gynecol 1961;81:281-290

6 Palma P, Dávila H, et al. Uroginecología. Confederación Americana de Urología. 2006:13-19

7 Ulmsten U, Henriksson L, Johnson P, Varhos G. An ambulatory surgical procedure under local anesthesia for treatment of female urinary incontinence. Int Urogynecol J Pelvic Floor Dysfunct 1996;7(02):81-85, discussion 85-86

8 Nilsson CG, Palva K, Rezapour M, Falconer C. Eleven years prospective follow-up of the tension-free vaginal tape procedure for treatment of stress urinary incontinence. Int Urogynecol J Pelvic Floor Dysfunct 2008;19(08):1043-1047
9 Ogah J, Cody JD, Rogerson L. Minimally invasive synthetic suburethral sling operations for stress urinary incontinence in women. Cochrane Database Syst Rev 2009;(04):CD006375

10 Levin I, Groutz A, Gold R, Pauzner D, Lessing JB, Gordon D. Surgical complications and medium-term outcome results of tension-free vaginal tape: a prospective study of 313 consecutive patients. Neurourol Urodyn 2004;23(01):7-9

11 Dmochowski RR, Blaivas JM, Gormley EA, et al; Female Stress Urinary Incontinence Update Panel of the American Urological Association Education and Research, Inc Update of AUA guideline on the surgical management of female stress urinary incontinence. J Urol 2010;183(05):1906-1914

12 Lapitan MC, Cody DJ, Grant AM. Open retropubic colposuspension for urinary incontinence in women. Cochrane Database Syst Rev 2005;(03):CD002912

13 Rehman H, Bezerra CCB, Bruschini H, Cody JD. Traditional suburethral sling operations for urinary incontinence in women. Cochrane Database Syst Rev 2011;(01):CD001754

14 Albo ME, Richter HE, Brubaker L, et al; Urinary Incontinence Treatment Network Burch colposuspension versus fascial sling to reduce urinary stress incontinence. N Engl J Med 2007;356(21): 2143-2155

15 Sistema de información de precios de medicamentos, SISMED. Listado de Precios Promedio y Unidades en la cadena de comercialización de Medicamentos -Enero a Diciembre de 2008. [Consultado enero 2012]. http://www.sispro.gov.co/SISMED/PDF/ Circular_2_2010/Publicacion_PreciosReportados_200801a200812. pdf

16 Consejo Directivo del Instituto de Seguros Sociales. Acuerdo 256 de 2001, Por el cual se aprueba el "Manual de tarifas" de la Entidad Promotora de Salud del Seguro Social "EPS-ISS". 2001 Dic. [Consultado enero 2012]. http://lexsaludcolombia.files.wordpress. com/2010/10/tarifas-iss-2001.pdf

17 http://datos.bancomundial.org/indicador/NY.GDP.PCAP.CD 